\title{
Template-Assisted Scalable Nanowire Networks
}

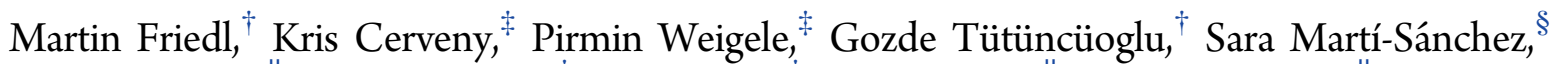
Chunyi Huang, ${ }^{\|}$Taras Patlatiuk, ${ }^{\ddagger}$ Heidi Potts, ${ }^{\dagger}$ Zhiyuan Sun, ${ }^{\| \odot}$ Megan O. Hill, ${ }^{\| \odot}$ Lucas Güniat, ${ }^{\dagger}$

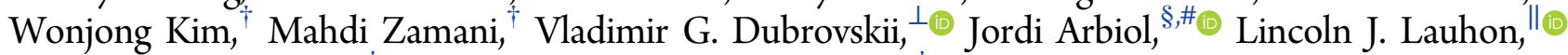
Dominik M. Zumbühl, ${ }^{\dagger}$ and Anna Fontcuberta i Morral*, ${ }^{*}$

\author{
${ }^{\dagger}$ Laboratoire des Matériaux Semiconducteurs, École Polytechnique Fédérale de Lausanne, EPFL, 1015 Lausanne, Switzerland \\ ${ }^{\ddagger}$ Department of Physics, University of Basel, Klingelbergstrasse 82, CH-4056 Basel, Switzerland \\ ${ }^{\S}$ Catalan Institute of Nanoscience and Nanotechnology (ICN2), CSIC and BIST, Campus UAB, Bellaterra, 08193 Barcelona, \\ Catalonia Spain \\ "Department of Materials Science and Engineering, Northwestern University, Evanston, Illinois 60208, United States \\ ${ }^{\perp}$ ITMO University, Kronverkskiy pr. 49, 197101 St. Petersburg, Russia \\ \#ICREA, Pg. Lluís Companys 23, 08010 Barcelona, Catalonia, Spain
}

Supporting Information

\begin{abstract}
Topological qubits based on Majorana Fermions have the potential to revolutionize the emerging field of quantum computing by making information processing significantly more robust to decoherence. Nanowires are a promising medium for hosting these kinds of qubits, though branched nanowires are needed to perform qubit manipulations. Here we report a gold-free templated growth of III-V nanowires by molecular beam epitaxy using an approach that enables patternable and highly regular branched nanowire arrays on a far greater scale than what has been reported thus

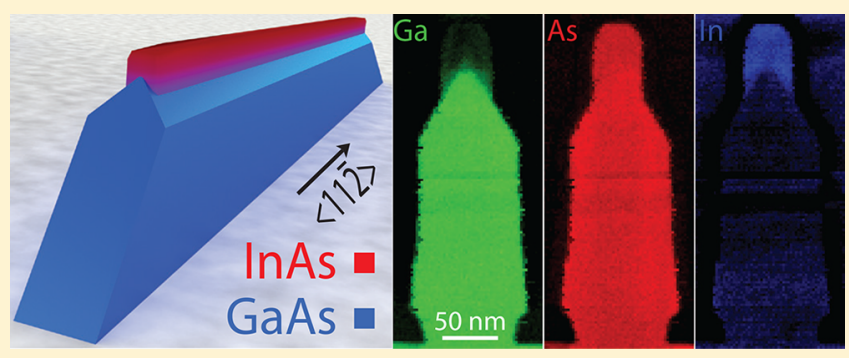
far. Our approach relies on the lattice-mismatched growth of InAs on top of defect-free GaAs nanomembranes yielding laterally oriented, low-defect InAs and InGaAs nanowires whose shapes are determined by surface and strain energy minimization. By controlling nanomembrane width and growth time, we demonstrate the formation of compositionally graded nanowires with cross-sections less than $50 \mathrm{~nm}$. Scaling the nanowires below $20 \mathrm{~nm}$ leads to the formation of homogeneous InGaAs nanowires, which exhibit phase-coherent, quasi-1D quantum transport as shown by magnetoconductance measurements. These results are an important advance toward scalable topological quantum computing.
\end{abstract}

KEYWORDS: InAs, nanowires, GaAs, nanoscale membranes, template-assisted, weak localization

$I^{n}$

$\mathrm{n}$ the past few years, much progress has been made toward fabricating and scaling up qubit density to build universal quantum computing systems that can outperform classical computers by quantum schemes. ${ }^{1-5}$ The ideal qubit should combine long coherence times, fast qubit manipulation, and small size, while maintaining scalability to many-qubit systems. Long coherence times are fundamentally challenging to achieve in various qubit systems due to the presence of numerous forms of environmental noise, requiring operating temperatures in the range of a hundred millikelvin." A system, which has been proposed to be much more robust against such perturbations, is the topological qubit. ${ }^{8,9}$ This type of qubit, for example, composed of Majorana Fermions (MFs) $)^{8,10}$ or parafermions (MPFs), ${ }^{11,12}$ would have the inherent property of being topologically protected and would thus exhibit exceptionally long coherence times. Signatures of MF states have been observed experimentally in, among other systems, III-V semiconductor nanowires (NWs) in close proximity to an swave superconductor, ${ }^{13-15}$ while a few other groups have reported anomalous MF signatures in similar systems. ${ }^{16-18}$ In general, these studies have focused on using III-V materials, such as InAs and InSb, due to their high-spin-orbit coupling strength and g-factor. ${ }^{19}$ Current efforts are focused on performing the first manipulations of MFs to further verify theoretical predictions, for which low-disorder, connected onedimensional (1D) branches are required. ${ }^{20}$ Gold-free and defect-free NW branches made of a high-purity, high-spinorbit III-V material would be an ideal platform for manipulating MFs. Excellent progress has been made toward this goal with reports on the growth of monocrystalline goldassisted InSb NW branches, which display a weak antilocalization due to the large spin-orbit interaction of the material, as well as a hard superconducting gap. ${ }^{21,22}$ Scalability is another

Received: February 7, 2018

Revised: $\quad$ March 23, 2018

Published: March 26, 2018 
important aspect of any future computational system, and on this front, the Riel group has recently demonstrated patternable ballistic InAs NW crosses through template-assisted growth on silicon. $^{23}$

Despite recent progress, a few challenges still exist with current methods to produce branched structures. The fabrication of NW networks and intersections has been explored for many years for classical computing by overlapping individual wires. $^{24-26}$ For MF applications, the stringent requirement of maintaining coherent transport across the intersection means that, currently, the most popular NW cross structures rely on the intersection of two gold-catalyzed NWs grown along two different $<111>\mathrm{B}$ directions leading to an interface-free junction. ${ }^{27-31}$ After growth, free-standing crosses are obtained, which then need to be transferred onto a separate substrate for further device fabrication, limiting the ultimate scalability. A scalable scheme would instead enable the NW growth and intersections to be realized directly on the final device substrate. At the same time, for future device integration, the use of gold seeds poses a problem for compatibility with CMOS technologies. ${ }^{32}$ Here, we demonstrate a new approach to grow gold-free branched $\mathrm{In}(\mathrm{Ga}) \mathrm{As}$ NWs at the wafer scale by using GaAs nanomembranes (NMs) as templates.

Defect-free GaAs NMs of exceptional quality constitute the ideal templates for further $\operatorname{In}(\mathrm{Ga})$ As NW growth. ${ }^{33,34}$ Such structures have been successfully grown by both metalorganic chemical vapor deposition (MOCVD) and molecular beam epitaxy (MBE) using a gold-free selective area approach. ${ }^{35,36}$ The NMs are patternable at the wafer scale and can additionally be fabricated in the form of Y-shaped structures by growing them along the three $\langle 11-2\rangle$ directions on GaAs (111) B substrates. ${ }^{35}$ When the growth of these GaAs NMs is followed by InAs, the InAs accumulates at the top of the NMs, forming In(Ga)As NWs along the NM vertex, as depicted schematically in Figure 1a. Shown in Figure $1 \mathrm{~b}$ is the progression of the NW growth, which initiates as InGaAs and then evolves to pure

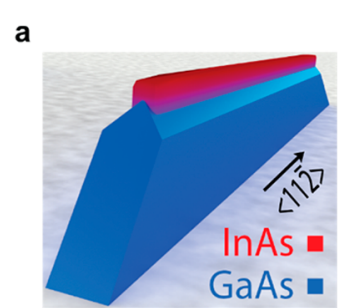

d

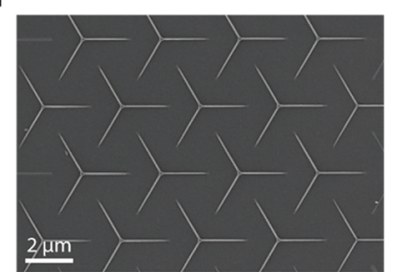

\section{b C}

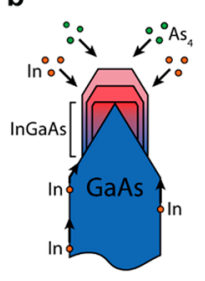

e

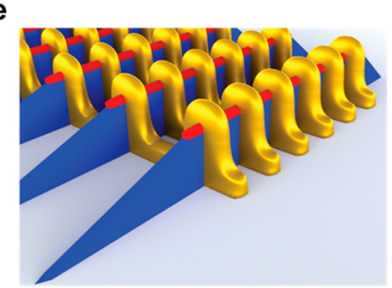

Figure 1. Growth of NWs on NMs. (a) Model of a single GaAs/InAs NM/NW structure. (b) Diagram showing NW growth progression. (c) A $30^{\circ}$ tilted scanning electron microscope (SEM) image of a branched NM/NW structure taken with an energy-selective backscattered electron detector for $\mathrm{z}$-contrast. The image is false-colored for visibility and annotated with relevant substrate directions. (d) SEM image of branched NW structures grown in a regular array. (e) Model of an array of contacted linear NM/NW structures used in magnetotransport measurements.
InAs for longer growth times. By varying the deposition times and growth conditions, the size and composition of the NWs can be changed. Combining the concepts of patterning NMs into Y-branches and performing $\operatorname{In}(\mathrm{Ga})$ As $\mathrm{NW}$ growth on top of GaAs NMs, Y-shaped $\operatorname{In}(\mathrm{Ga})$ As NW junctions can be obtained, as shown in Figure 1c,d. Our approach thereby enables the growth of gold-free branched NWs at the wafer scale.

In this Letter, as the first step toward building MF devices based on this approach, we demonstrate the growth of lowdefect linear In(Ga)As NWs based on GaAs NMs. Magnetotransport measurements (depicted schematically in Figure 1e) demonstrate weak localization in the diffusive regime suggesting quasi-1D quantum transport. This makes such NWs ideal candidates for future quantum computing schemes.

Nanomembranes as a Platform. Membranes with top ridges parallel to the substrate were grown as described previously. ${ }^{36}$ The InAs NWs were then grown for 200s at an $\mathrm{As}_{4}$ flux of $8 \times 10^{-6} \mathrm{Torr}$, an In rate of $0.2 \AA / \mathrm{s}$ and a substrate temperature of $540{ }^{\circ} \mathrm{C}$. This optimized substrate temperature yielded continuous InAs growth on top of the NMs. The details can be found in the Supporting Information. A Si dopant flux of $\sim 10^{13}$ atoms $/ \mathrm{cm}^{2} / \mathrm{s}$ was also introduced to increase the conductivity of the NWs.

The NW morphology, composition, and structural quality were extensively characterized by correlated analysis using various electron microscopy techniques. These included electron energy loss spectroscopy (EELS) and atomicresolution aberration-corrected annular dark field scanning transmission electron microscopy (ADF-STEM). The results were then coupled with geometrical phase analysis (GPA) to give strain information, ${ }^{37,38}$ which in turn was fed into a semiempirical model to understand the formation of the NWs from a theoretical standpoint.

Analysis of focused ion-beam (FIB) lamellas prepared perpendicular to the NW axis by ADF-STEM and correlated EELS (Figure $2 \mathrm{a}-\mathrm{c}$ ) show that $\sim 50 \mathrm{~nm}$ diameter InAs NWs form on the $250 \mathrm{~nm}$ tall GaAs NMs. The InAs material preferentially accumulates along the top ridge of the GaAs NM, forming the NW, which is primarily InAs with a $20 \mathrm{~nm}$ thick intermixed InGaAs region at its base. (See the Supporting Information for details.) This InGaAs region likely occurs due to strain-mediated intermixing with the GaAs NM below as has been observed in InAs quantum dots on GaAs. ${ }^{39}$ It is important to note that we observe no In signal from the NMs; the faint signal seen in the EELS map is believed to be created during the FIB cutting by a combination of redeposition of the $\mathrm{TiO}_{x}$ protective layer and surface diffusion of the highly mobile In adatoms. Looking instead at the NW facets, as seen in Figure $2 \mathrm{~d}$, the resulting InAs NW structures are terminated by two (110) facets on the sides and have a single flat (111) top facet. The appearance of this (111) facet, instead of the two $\{113\}$ facets as in the GaAs NMs, can be explained by the higher $\mathrm{As}_{4}$ flux used in the InAs NW growth. ${ }^{40,41}$

No defects were observed when viewing this transverse lamella in atomic-resolution ADF-STEM mode. As strain along the NW axis was predicted to be more difficult to relax than in the transverse direction, a second FIB lamella was prepared parallel to the axis of the NW and also imaged using atomicresolution ADF-STEM. Here, a few misfit dislocations were observed near the InAs/GaAs interface, with an estimated density of approximately $100 / \mu \mathrm{m}$, as described in the Supporting Information. This constitutes a $40 \%$ reduction in 
a $\quad$ b

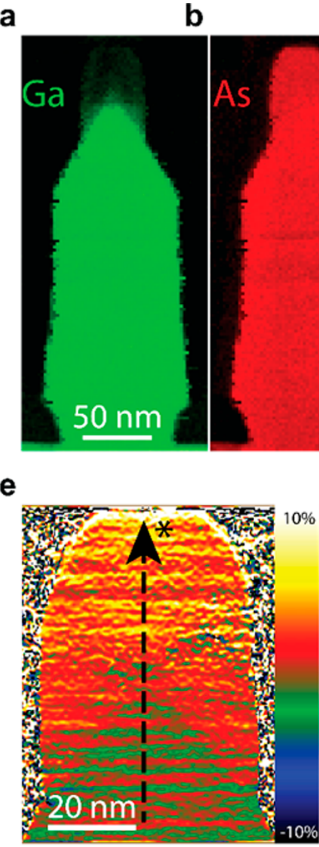

g

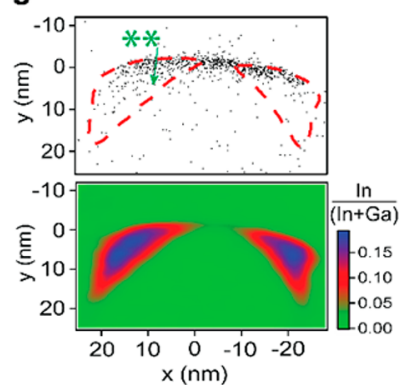

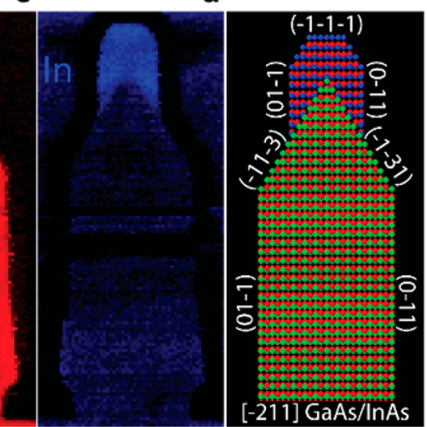

f

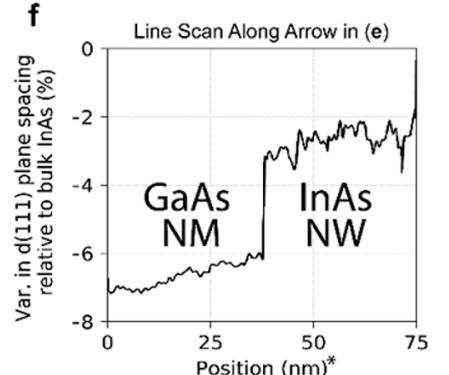

h

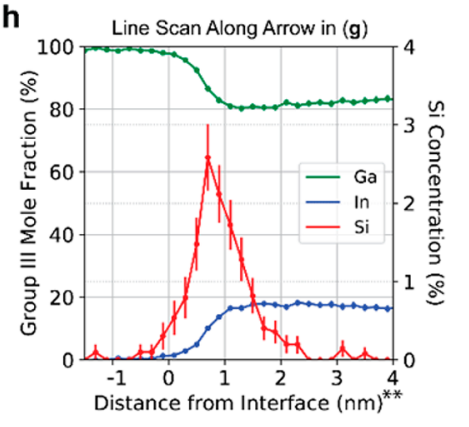

Figure 2. TEM and APT analysis. (a-c) EELS maps with elemental contrast of a NM/NW cross section. Note that the overlap of the In EELS signal with the $\mathrm{Ti}$ signal from the $\mathrm{TiO}_{x}$ capping layer has caused an anomalous background of In within the NM, which is not physical. (d) Atomic model showing faceting of the NM/NW heterostructure. (e) GPA map of the InAs NW region. (f) Line scan along arrow in panel e. (g) APT map of scaled-down NWs used in electrical measurements showing In concentration (lower map) and accumulation of Si dopant atoms (black dots) at the surface of the NW with In isoconcentration lines as a guide to the eye (upper map). Note that, since the NWs were capped with GaAs for APT analysis, the measured In concentration can be considered as a lower bound for the uncapped structures. (h) Proxigram line scan along the dashed arrow in panel g showing $\mathrm{Si}$ accumulation on the NW top facet and In concentration within the NW.

dislocation density with respect to the equivalent $2 \mathrm{D}$ growth of InAs on GaAs and at least three times improvement with respect to the twin density typically observed in self-catalyzed InAs NWs. ${ }^{42-44}$

Turning now to explaining the morphology of the structures, an analytical model shows that the surface and strain energy minimization play the most important role in driving the NW to adopt the observed shape (Supporting Information). By taking advantage of the atomic resolution offered by ADFSTEM images, GPA was performed on the initial FIB lamella cut perpendicular to the NW axis. Looking specifically at the (111) plane spacing, a substantial 2-3\% residual compressive strain is observed within the NW, as shown in Figure 2e, with the corresponding line scan given in Figure $2 \mathrm{f}$. Using this strain value, a semiquantitative model was developed to describe the NW formation energy, taking into account the InAs/GaAs surface energies and the InAs elastic strain energy. The total energy of the system was then minimized with respect to the NW aspect ratio. Interestingly, the experimentally observed aspect ratio coincides with that of the theoretical minimum energy shape, suggesting that the NW shape is simply driven by energy minimization. (See the Supporting Information for details.)

Electrical Transport in the Mesoscopic Regime. To bring the NWs into the $1 \mathrm{D}$ electrical transport regime, they were downscaled to $\sim 20 \mathrm{~nm}$ diameters by narrowing the GaAs NMs by using smaller $\mathrm{SiO}_{2}$ openings and shorter growth times for less lateral growth. The resulting NWs were smaller both laterally and vertically, resulting in intermixed InGaAs NWs, as depicted for small diameter NWs in the growth progression diagram in Figure $1 \mathrm{~b}$. These results were confirmed by performing atom probe tomography (APT), which additionally yielded information about the Si dopant distribution.

The APT analysis confirmed the presence of an InGaAs NW, while also uncovering signs of dopant rejection during crystal growth, causing an accumulation of Si atoms at the NW surface. Figure $2 \mathrm{~g}$ shows a typical APT map of the In mole fraction and $\mathrm{Si}$ dopant distribution. A quantitative composition profile of the NW surface was extracted in the proximity histogram (proxigram) shown in Figure $2 \mathrm{~h}$. In this sample, the NW group III mole fractions are $\sim 17 \%$ In and $\sim 83 \% \mathrm{Ga}$. (Additional maps are given in the Supporting Information.) Analysis of different InGaAs samples by APT tomography under similar conditions shows a slight tendency toward preferential In evaporation. In addition, a GaAs capping layer was deposited on the APT sample after the NW growth, which may have enhanced the Ga intermixing. ${ }^{45}$ For these reasons, we consider the In mole fraction in this NW as a lower bound. Although a Si flux was present during InAs NW growth, the Si atoms are not homogeneously distributed throughout the NW (Figure $2 \mathrm{~g}$ ). The $\mathrm{Si}$ atoms instead appear to accumulate preferentially at the (111) growth interface, resulting in a quasiremotely doped structure.

The electrical properties of the NWs were explored through multi-contact resistance measurements on an array of NWs, for which an example device is shown in Figure 3a. An array of 34 NWs, comparable to those on which APT was performed, was used for these tests as a way to obtain the average response from many devices. Standard four-point measurements were then carried out at room and low temperatures before moving to low-temperature magnetoconductance transport experiments.

Room-temperature transmission line measurements, shown in Figure $3 \mathrm{~b}$, gave linearly scaling and repeatable resistances, suggesting that a good-quality contact was achieved. A control sample without InAs NWs (as shown in green in Figure 3b) shows a 5 orders of magnitude increase in resistance, directly proving that the observed conduction occurs due to the InAs deposition. Device behavior remained linear and ohmic down to $4.2 \mathrm{~K}$, albeit with an increased contact resistance.

Magnetoconductance measurements at $1.5 \mathrm{~K}$ revealed a zerofield minimum of conductance consistent with a weak localization (WL) behavior, as shown in Figure $3 c^{46}$ The conductance of the devices was measured under constant bias, while the magnetic field was applied perpendicular to the substrate and was swept from $-8 \mathrm{~T}$ to $8 \mathrm{~T}$. This analysis indicates conduction in the quasi-1D transport regime and 


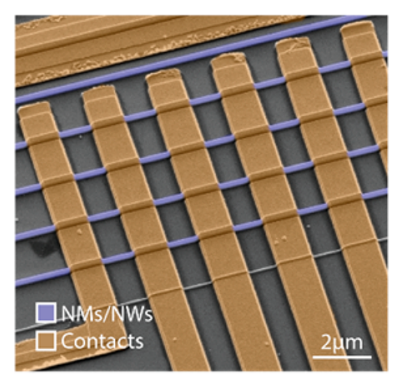

b

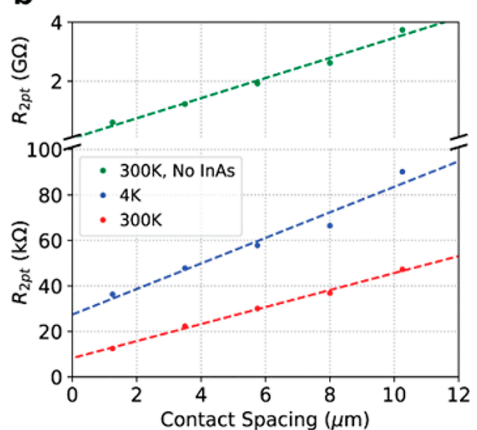

C

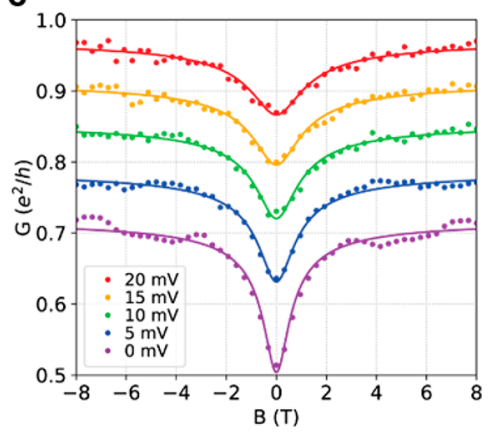

Figure 3. Magnetotransport measurements. (a) False-colored SEM image of four InAs/GaAs NW/NMs contacted in parallel. (b) Transmission line measurement to extract contact resistance and resistivity (per NW) at 4 and $300 \mathrm{~K}$, in comparison with NMs without the InAs deposition step. (c) Average differential conductance per NW as a function of magnetic field perpendicular to the NWs for a range of bias voltages measured at $1.5 \mathrm{~K}$ for a $1.25 \mu \mathrm{m}$ long NW segment. The traces for biases above $0 \mathrm{mV}$ are offset for clarity.

elucidates important quantum figures of merit such as coherence length and spin-orbit length, as described below.

A coherence length, $l_{\varphi}$, of $130 \mathrm{~nm}$ and a lower bound on the spin-orbit length, $l_{\mathrm{so}}$, of $280 \mathrm{~nm}$ were obtained by fitting the experimental data. The system was assumed to be in the diffusive regime with the electron mean free path $l_{\mathrm{e}} \ll W$, with $W$ being the width of the conducting channel, estimated from the APT results to be about $20 \mathrm{~nm}$. In this regime, $l_{\mathrm{e}}$ is thus constrained to be a few nanometers due to the large amount of dopant and surface scattering. A simple quasi-1D model for the quantum corrections to the conductivity in the diffusive limit (details in Methods) gives an excellent agreement with the data and yields $l_{\varphi} \sim 130 \pm 4 \mathrm{~nm}$ near zero bias, as shown in the solid traces in Figure 3c. In the absence of weak antilocalization, and adding spin-orbit coupling to the model, a lower bound for $l_{\text {so }}$ of $280 \mathrm{~nm}$ is estimated. Some small variations in the conductance at large magnetic fields are noticeable, presumably signatures of conductance fluctuations, which are strongly suppressed due to averaging from the parallel NW arrangement as well as the relatively short coherence length.

These initial results show that the electron confinement at the top of NWs grown on NMs is sufficient to produce quasi$1 \mathrm{D}$ conduction. These NWs could therefore be viable hosts for Majorana bound states provided that two potential obstacles are addressed. First, a NW made of a higher spin-orbit material such as pure or nearly pure InAs or InSb will be required; i.e., intermixing should be reduced or eliminated. This will be apparent by the observation of weak antilocalization instead of weak localization. Second, impurity, interface, and alloy scattering need to be reduced such that $l_{\mathrm{e}}$ transitions from the diffusive to the ballistic transport regime.

Conclusion and Outlook. The fast-growing field of quantum computing and the promise of robust, topologically protected qubits with III-V NWs drives the pursuit of scalable approaches to branched NW networks. We have described a path forward using GaAs NMs as templates for the $\operatorname{In}(\mathrm{Ga})$ As NW growth. By exploiting strain in the highly mismatched InAs/GaAs system, continuous, low-defect NWs were formed. We have further observed weak localization, demonstrating that such NWs can provide sufficient confinement to achieve quasi1D conduction. Our gold-free wafer-scale approach to branched NWs serves as a platform for future investigations into $1 \mathrm{D}$ transport and quantum computation with III-V NW networks with many exciting possibilities. From the MBE growth perspective, using GaSb NMs already described by other groups to grow $\mathrm{InSb} \mathrm{NWs}$ would be interesting due to the higher g-factor of InSb. ${ }^{19,47}$ Alternatively, suitable plastic strain relaxation, for example, by interfacial misfit array formation, ${ }^{48}$ may enable GaAs NMs to be viable templates for InSb NW growth. At the same time, the growth of new kinds of structures with additional functionalities is another avenue to explore, including, for example, research into parafermion devices by stacking multiple NWs on top of each other. ${ }^{12}$ The wealth of intriguing new possibilities and approaches offered by templateassisted III-V NW growth makes this method an important step toward realizing a scalable quantum computing scheme based on NW topological qubits.

Methods. Substrate Preparation. Undoped GaAs (111) B substrates were prepared by first depositing $25 \mathrm{~nm}$ of $\mathrm{SiO}_{2}$ by the plasma enhanced chemical vapor deposition (PECVD). This was followed by e-beam lithography using ZEP resist and low-temperature development to achieve low line edge roughness. ${ }^{49}$ Subsequent dry etching with fluorine chemistry was used to etch the $\mathrm{SiO}_{2}$ down to the GaAs surface, and a final wet etch in a dilute buffered HF solution helped remove any remaining oxide. This yielded openings varying from 30 to 100 $\mathrm{nm}$ in width and $10-20 \mu \mathrm{m}$ in length, depending on the ebeam pattern.

Growth. The nanostructures were grown in a DCA D600 Gen II solid-source MBE. The optimal growth of the GaAs/ InAs $\mathrm{NM} / \mathrm{NW}$ heterostructures was found to be at a temperature of $630{ }^{\circ} \mathrm{C} / 540{ }^{\circ} \mathrm{C}$ (as measured by the pyrometer), As flux of $4 \times 10^{-6} / 8 \times 10^{-6}$ Torr and Ga/In deposition rates of $1.0 / 0.2 \AA / \mathrm{s}$, respectively. The NMs were typically grown for $30 \mathrm{~min}$ (180 nm nominal $2 \mathrm{D}$ thickness), while the InAs NWs were grown for 200s (4 nm nominal 2D thickness).

(S)TEM. The cross sections of the NMs were prepared by focused ion-beam (FIB) milling normal to the substrate surface and investigated by atomic-resolution aberration-corrected annular dark field scanning transmission electron microscopy (ADF-STEM) in a probe corrected FEI Titan 60-300 keV microscope operated at $300 \mathrm{keV}$. The elemental maps were obtained by using an electron energy loss spectrometer (EELS) coupled to a Tecnai F20 microscope.

Contacting. Contacts were patterned by e-beam lithography followed by dual-angle evaporation of 14/80 nm of $\mathrm{Cr} / \mathrm{Au}$ for good side-wall coverage. Before metallization, an $\mathrm{O}_{2}$ plasma clean and a $6 \mathrm{~min}$ ammonium polysulfide etch at $40{ }^{\circ} \mathrm{C}$ were used to ensure a clean, oxide-free contact. ${ }^{50}$ 
Quantum Transport Model. The conductance of the NW is described as

$$
\Delta G=-\frac{2 \mathrm{e}^{2}}{h L}\left(\frac{1}{l_{\varphi}^{2}}+\frac{1}{l_{B}^{2}}\right)^{-1 / 2}
$$

where $L$ is the spacing between the contacts and $l_{B}$ is the magnetic dephasing length given by $l_{B}=\sqrt{D \tau_{B}}$, with $D$ as the diffusion constant $\left(D_{1 \mathrm{D}}=v_{F} l_{e}\right.$ for $1 \mathrm{D}$ diffusion $)$. In this limit, the magnetic dephasing time $\tau_{B}$ is given by $\tau_{B}=3 l_{\mathrm{m}}^{4} / W^{2} D$, where $l_{\mathrm{m}}=\sqrt{\hbar / e B}$ is the magnetic length. ${ }^{46,51,52}$

GaAs Capping. After the growth of the NW/NM heterostructures, a $\sim 30 \mathrm{~nm}$ GaAs cap was deposited in situ by $\mathrm{MBE}$ at $400{ }^{\circ} \mathrm{C}$. In the middle of this GaAs cap, the In shutter was opened for $10 \mathrm{~s}$, making a few-monolayer insertion of $\mathrm{In}_{0.16} \mathrm{Ga}_{0.84} \mathrm{As}$, indicating the midpoint of the GaAs cap. The $\mathrm{NW} / \mathrm{NM}$ heterostructures were then coated with a $\sim 110 \mathrm{~nm}$ GaAs layer ${ }^{53}$ using ion-beam sputtering at $9 \mathrm{kV}$ and $7.5 \mathrm{~mA}$ for $1 \mathrm{~h}$. The capping layer protected the sample from damage caused by the ion beam during FIB.

APT. A standard lift-out method ${ }^{53,54}$ was performed in a FEI Helios dual-beam focused ion-beam (FIB) microscope with a micromanipulator, and the as-prepared wedge-shaped samples were welded onto Si microposts. Finally, the needle-shaped APT specimens were obtained by ion-beam annular milling. APT was performed with a local-electrode atom-probe (LEAP) 4000X Si tomograph (Cameca, Madison, WI) at a sample temperature of $40 \mathrm{~K}$ and a background pressure of $3 \times 10^{-11}$ Torr. An ultraviolet focused laser with a wavelength of $355 \mathrm{~nm}$ was used to evaporate the sample atoms into ions, at a pulse rate of $250 \mathrm{kHz}$ and detection rate of $0.7 \%$. The pulse energy was gradually changed from $1.2 \mathrm{pJ}$ to $0.8 \mathrm{pJ}$ during the evaporation process. The data was reconstructed using IVAS 3.8.1 to provide a $3 \mathrm{D}$ composition profile. SEM images of the nanotips taken in the FIB were used to guide the choices of the reconstruction parameters.

\section{ASSOCIATED CONTENT}

\section{S Supporting Information}

The Supporting Information is available free of charge on the ACS Publications website at DOI: 10.1021/acs.nanolett.8b00554.

InAs growth studies, Raman spectroscopy, strain analysis, growth model, change in free energy of formation with aspect ratio, NW composition, InAs crystal quality, defect density, magnetotransport model, conductivity map, and APT mass spectra (PDF)

\section{AUTHOR INFORMATION}

\section{Corresponding Author}

*E-mail: anna.fontcuberta-morral@epfl.ch.

\section{ORCID}

Zhiyuan Sun: 0000-0003-3981-9083

Megan O. Hill: 0000-0002-7663-7986

Vladimir G. Dubrovskii: 0000-0003-2088-7158

Jordi Arbiol: 0000-0002-0695-1726

Lincoln J. Lauhon: 0000-0001-6046-3304

Anna Fontcuberta i Morral: 0000-0002-5070-2196

\section{Notes}

The authors declare no competing financial interest.

\section{ACKNOWLEDGMENTS}

Authors from EPFL and U. Basel acknowledge funding through the NCCR QSIT. Authors from EPFL further thank funding from SNF (project no. IZLRZ2-163861) and H2020 via the ITN project INDEED. Work at U. Basel was partially supported by the Swiss NSF, and the Swiss Nanoscience Institute SNI. S.M.S. acknowledges funding from "Programa Internacional de Becas "la Caixa"-Severo Ochoa". J.A. and S.M.S. acknowledge funding from Generalitat de Catalunya 2017 SGR 327 and the Spanish MINECO coordinated project ValPEC (ENE2017-85087-C3). ICN2 acknowledges support from the Severo Ochoa Programme (MINECO, grant no. SEV2013-0295) and is funded by the CERCA Programme/ Generalitat de Catalunya. This work has received funding from the European Union's Horizon 2020 Research and Innovation Programme under grant agreement no. 654360 NFFA-Europe. Part of the present work has been performed in the framework of Universitat Autònoma de Barcelona Materials Science Ph.D. program. The atomic-resolution ADF-STEM microscopy was conducted in the Laboratorio de Microscopias Avanzadas at the Instituto de Nanociencia de AragonUniversidad de Zaragoza. J.A. and S.M.S. thank them for offering access to their instruments and expertise. V.G.D. thanks the Ministry of Education and Science of the Russian Federation for financial support under grant no. 14-613-210055 (project ID RFMEFI61316 × 0055). L.J.L. acknowledges support of NSF DMR-1611341. M.O.H. acknowledges support of the NSF GRFP. Atom-probe tomography was performed at the Northwestern University Center for Atom-Probe Tomography (NUCAPT). The LEAP tomograph at NUCAPT was purchased and upgraded with grants from the NSF-MRI (DMR-0420532) and ONR-DURIP (N00014-0400798, N00014-0610539, N00014-0910781, and N00014-1712870) programs. NUCAPT received support from the MRSEC program (NSF DMR-1720139) at the Materials Research Center, the SHyNE Resource (NSF ECCS-1542205), and the Initiative for Sustainability and Energy (ISEN) at Northwestern University.

\section{REFERENCES}

(1) Loss, D.; DiVincenzo, D. P. Quantum Computation with Quantum Dots. Phys. Rev. A: At., Mol., Opt. Phys. 1998, 57, 120.

(2) Kielpinski, D.; Monroe, C.; Wineland, D. J. Architecture for a large-scale ion-trap quantum computer. Nature 2002, 417, 709-711.

(3) Gottesman, D. An Introduction to Quantum Error Correction and Fault-Tolerant Quantum Computation. Proc. Symp. Appl. Math. 2010, 68, 13-46.

(4) Reilly, D. J. Engineering the quantum-classical interface of solidstate qubits. npj Quantum Inf. 2015, 1, 15011.

(5) Gambetta, J. M.; Chow, J. M.; Steffen, M. Building logical qubits in a superconducting quantum computing system. npj Quantum Inf. 2017, 3, 2.

(6) Takahashi, S.; et al. Decoherence in crystals of quantum molecular magnets. Nature 2011, 476, 76-79.

(7) Goetz, J.; et al. Second-order decoherence mechanisms of a transmon qubit probed with thermal microwave states. Quantum Sci. Technol. 2017, 2, 025002.

(8) Kitaev, A. Y. Fault-tolerant quantum computation by anyons. Ann. Phys. (Amsterdam, Neth.) 2003, 303, 2-30.

(9) Stern, A.; Lindner, N. H. Topological Quantum ComputationFrom Basic Concepts to First Experiments. Science (Washington, DC, U. S.) 2013, 339, 1179-1184.

(10) Kitaev, A. Y. Unpaired Majorana fermions in quantum wires. Physics-Uspekhi 2001, 44, 131-136. 
(11) Klinovaja, J.; Loss, D. Parafermions in an Interacting Nanowire Bundle. Phys. Rev. Lett. 2014, 112, 246403.

(12) Klinovaja, J.; Loss, D. Time-reversal invariant parafermions in interacting Rashba nanowires. Phys. Rev. B: Condens. Matter Mater. Phys. 2014, 90, 1-8.

(13) Mourik, V.; et al. Signatures of Majorana Fermions in Hybrid Superconductor-Semiconductor Nanowire Devices. Science (Washington, DC, U. S.) 2012, 336, 1003-1007.

(14) Das, A.; et al. Zero-bias peaks and splitting in an Al-InAs nanowire topological superconductor as a signature of Majorana fermions. Nat. Phys. 2012, 8, 887-895.

(15) Albrecht, S. M.; et al. Exponential Protection of Zero Modes in Majorana Islands. Nature 2016, 531, 206-209.

(16) Deng, M. T.; et al. Anomalous zero-bias conductance peak in a $\mathrm{Nb}-\mathrm{InSb}$ nanowire-Nb hybrid device. Nano Lett. 2012, 12, 6414-6419.

(17) Lee, Y.-H.; et al. Synthesis of large-area MoS2 atomic layers with chemical vapor deposition. Adv. Mater. 2012, 24, 2320-5.

(18) Lee, E. J. H.; et al. Spin-resolved Andreev levels and parity crossings in hybrid superconductor-semiconductor nanostructures. Nat. Nanotechnol. 2014, 9, 79-84.

(19) Lutchyn, R. M. Realizing Majorana zero modes in superconductor-semiconductor heterostructures. arXiv 2017, 1-18.

(20) Alicea, J.; Oreg, Y.; Refael, G.; von Oppen, F.; Fisher, M. P. a. Non-Abelian statistics and topological quantum information processing in 1D wire networks. Nat. Phys. 2011, 7, 412-417.

(21) Gazibegovic, S.; et al. Epitaxy of advanced nanowire quantum devices. Nature 2017, 548, 434-438.

(22) Plissard, S. R.; et al. Formation and electronic properties of InSb nanocrosses. Nat. Nanotechnol. 2013, 8, 859-864.

(23) Gooth, J.; et al. Ballistic One-Dimensional InAs Nanowire Cross-Junction Interconnects. Nano Lett. 2017, 17, 2596-2602.

(24) Huang, Y.; Duan, X.; Wei, Q.; Lieber, C. M. Directed assembly of one-dimensional nanostructures into functional networks. Science (Washington, DC, U. S.) 2001, 291, 630-633.

(25) Huang, Y.; et al. Logic gates and computation from assembled nanowire building blocks. Science (Washington, DC, U. S.) 2001, 294, 1313-1317.

(26) Melosh, N. A.; et al. Ultrahigh-density nanowire lattices and circuits. Science (Washington, DC, U. S.) 2003, 300, 112-115.

(27) Gül, Ö.; et al. Towards high mobility InSb nanowire devices. Nanotechnology 2015, 26, 215202.

(28) Car, D.; Wang, J.; Verheijen, M. A.; Bakkers, E. P. A. M.; Plissard, S. R. Rationally Designed Single-Crystalline Nanowire Networks. Adv. Mater. 2014, 26, 4875-4879.

(29) Rieger, T.; et al. Crystal Phase Transformation in SelfAssembled InAs Nanowire Junctions on Patterned Si Substrates. Nano Lett. 2016, 16, 1933-1941.

(30) Kang, J. H.; et al. Crystal structure and transport in merged InAs nanowires MBE grown on (001) InAs. Nano Lett. 2013, 13, 51905196.

(31) Dalacu, D.; Kam, A.; Austing, D. G.; Poole, P. J. Droplet dynamics in controlled InAs nanowire interconnections. Nano Lett. 2013, 13, 2676-2681.

(32) Collins, C. B.; Carlson, R. O.; Gallagher, C. J. Properties of golddoped silicon. Phys. Rev. 1957, 105, 1168-1173.

(33) Tutuncuoglu, G.; et al. Towards defect-free 1-D GaAs/AlGaAs heterostructures based on GaAs nanomembranes. Nanoscale 2015, 7, 19453-60.

(34) Yang, Z.; et al. Revealing Large-Scale Homogeneity and Trace Impurity Sensitivity of GaAs Nanoscale Membranes. Nano Lett. 2017, 17, 2979-2984.

(35) Chi, C.-Y.; et al. Twin-Free GaAs Nanosheets by Selective Area Growth: Implications for Defect-Free Nanostructures. Nano Lett. 2013, 13, 2506-2515.

(36) Tutuncuoglu, G.; et al. Towards defect-free 1-D GaAs/AlGaAs heterostructures based on GaAs nanomembranes. Nanoscale 2015, 7, 19453-19460.
(37) de la Mata, M.; Magén, C.; Caroff, P.; Arbiol, J. Atomic Scale Strain Relaxation in Axial Semiconductor III-V Nanowire Heterostructures. Nano Lett. 2014, 14, 6614-6620.

(38) de la Mata, M.; et al. Twin-Induced InSb Nanosails: A Convenient High Mobility Quantum System. Nano Lett. 2016, 16, $825-833$.

(39) Lipinski, M. O.; Schuler, H.; Schmidt, O. G.; Eberl, K.; JinPhillipp, N. Y. Strain-induced material intermixing of InAs quantum dots in GaAs. Appl. Phys. Lett. 2000, 77, 1789.

(40) Pehlke, E.; Moll, N.; Scheffler, M. The Equilibrium Shape of Quantum Dots. arXiv 1996, 1-10.

(41) Moll, N.; Scheffler, M.; Pehlke, E. Influence of surface stress on the equilibrium shape of strained quantum dots. Phys. Rev. B: Condens. Matter Mater. Phys. 1998, 58, 4566-4571.

(42) Belk, J. G.; et al. Surface atomic configurations due to dislocation activity in InAs/GaAs(110) heteroepitaxy. Phys. Rev. B: Condens. Matter Mater. Phys. 1997, 56, 10289-10296.

(43) Nosho, B. Z.; Zepeda-Ruiz, L. A.; Pelzel, R. I.; Weinberg, W. H.; Maroudas, D. Surface morphology in InAs/GaAs(111)A heteroepitaxy: Experimental measurements and computer simulations. Appl. Phys. Lett. 1999, 75, 829-831.

(44) Potts, H.; et al. From Twinning to Pure Zincblende CatalystFree $\operatorname{InAs}(\mathrm{Sb})$ Nanowires. Nano Lett. 2016, 16, 637-643.

(45) García, J. M.; et al. Intermixing and shape changes during the formation of InAs self-assembled quantum dots. Appl. Phys. Lett. 1997, 71, 2014-2016.

(46) Beenakker, C. W. J.; van Houten, H. Boundary scattering and weak localization of electrons in a magnetic field. Phys. Rev. B: Condens. Matter Mater. Phys. 1988, 38, 3232-3240.

(47) Fahed, M.; Desplanque, L.; Troadec, D.; Patriarche, G.; Wallart, $\mathrm{X}$. Selective area heteroepitaxy of GaSb on GaAs $(001)$ for in-plane InAs nanowire achievement. Nanotechnology 2016, 27, 505301.

(48) Trampert, A.; Tournie, E.; Ploog, K. H. Influence of the growth mode on the microstructure of highly mismatch InAs/GaAs heterostructures. Physcia Status Solidi (a) 1994, 145, 481-489.

(49) Ocola, L. E.; Stein, A. Effect of cold development on improvement in electron-beam nanopatterning resolution and line roughness. J. Vac. Sci. Technol. B Microelectron. Nanom. Struct. 2006, 24, 3061.

(50) Suyatin, D. B.; Thelander, C.; Björk, M. T.; Maximov, I.; Samuelson, L. Sulfur passivation for ohmic contact formation to InAs nanowires. Nanotechnology 2007, 18, 105307.

(51) Hikami, S.; Larkin, A. I.; Nagaoka, Y. Spin-Orbit Interaction and Magnetoresistance in the Two Dimensional Random System. Prog. Theor. Phys. 1980, 63, 707-710.

(52) Al'tshuler, B.; Aronov, A. Magnetoresistance of thin films and of wires in a longitudinal magnetic field. JETP Lett. 1981, 33, 499.

(53) Sun, Z.; Hazut, O.; Yerushalmi, R.; Lauhon, L. J.; Seidman, D. $\mathrm{N}$. Criteria and considerations for preparing atom-probe tomography specimens of nanomaterials utilizing an encapsulation methodology. Ultramicroscopy 2018, 184, 225-233.

(54) Thompson, K.; et al. In situ site-specific specimen preparation for atom probe tomography. Ultramicroscopy 2007, 107, 131-139. 